\title{
Plano nacional de vacinação contra a COVID-19: uso de inteligência artificial espacial para superação de desafios
}

\author{
National COVID-19 vaccination plan: using artificial spatial \\ intelligence to overcome challenges in Brazil
}

Thiago Augusto Hernandes Rocha (https://orcid.org/0000-0002-6262-3276) ${ }^{1}$

Ghabriela Moura Boitrago (https://orcid.org/0000-0002-3710-6437) ${ }^{2}$

Rayanne Barbosa Mônica (https://orcid.org/0000-0003-0129-8382) ${ }^{3}$

Dante Grapiuna de Almeida (https://orcid.org/0000-0003-4434-0667) 4

Núbia Cristina da Silva (https://orcid.org/0000-0002-0809-2152) ${ }^{1}$

Débora Marcolino Silva (https://orcid.org/0000-0001-5126-6835) ${ }^{3}$

Sandro Haruyuki Terabe (https://orcid.org/0000-0003-1315-6226) ${ }^{5}$

Catherine Staton (https://orcid.org/0000-0002-6468-2894) ${ }^{1}$

Luiz Augusto Facchini (https://orcid.org/0000-0002-5746-5170) ${ }^{6}$

João Ricardo Nickenig Vissoci (https://orcid.org/0000-0001-7276-0402) ${ }^{1}$

${ }^{1}$ Duke Global Health Institute, Duke University, Global Emergency

Medicine Innovation and Implementation Research.

310, Trent Drive, Durham North Carolina USA.

rochahernandes3@

gmail.com

${ }^{2}$ Organizacão Pan-

Americana da Saúde

Organização Mundial da

Saúde. Brasília DF Brasil.

${ }^{3}$ Universidade de Brasília.

Brasília DF Brasil.

${ }^{4}$ Medomai Informática

Ltda. Belo Horizonte MG

Brasil.

${ }^{5}$ Escola Nacional de Saúde Pública Sergio Arouca,

Fundação Oswaldo Cruz.

Rio de Janeiro RJ Brasil.

${ }^{6}$ Departamento de Medicina

Social, Faculdade de

Medicina, Universidade

Federal de Pelotas. Pelotas

RS Brasil.

\begin{abstract}
This article explores the use of spatial artificial intelligence to estimate the resources needed to implement Brazil's COVID-19 immu nization campaign. Using secondary data, we conducted a cross-sectional ecological study adop ting a time-series design. The unit of analysis was Brazil's primary care centers (PCCs). A four-step analysis was performed to estimate the popula tion in PCC catchment areas using artificial in telligence algorithms and satellite imagery. We also assessed internet access in each PCC and con ducted a space-time cluster analysis of trends in cases of SARS linked to COVID-19 at municipal level. Around 18\% of Brazil's elderly population live more than 4 kilometer from a vaccination point. A total of 4,790 municipalities showed an upward trend in SARS cases. The number of PCCs located more than 5 kilometer from cell towers was largest in the North and Northeast regions. Innovative stra tegies are needed to address the challenges posed by the implementation of the country's National COVID-19 Vaccination Plan. The use of spatial artificial intelligence-based methodologies can help improve the country's COVID-19 response.
\end{abstract}

Key words Spatial analysis, Artificial intelligen ce, Mass vaccination, Immunization programs, Geographic mapping
Resumo O objetivo deste artigo é analisar o uso da inteligência artificial espacial no contexto da imunização contra COVID-19 para a seleção adequada dos recursos necessários. Trata-se de estudo ecológico de caráter transversal baseado em uma abordagem espaço-temporal utilizando dados secundários, em Unidades Básicas de Saúde do Brasil. Foram adotados quatro passos analíticos para atribuir um volume de população por unidade básica, aplicando algoritmos de inteligência artificial a imagens de satélite. Em paralelo, as condições de acesso à internet móvel e o mapeamento de tendências espaço-temporais de casos graves de COVID-19 foram utilizados para caracterizar cada município do país. Cerca de $18 \%$ da população idosa brasileira está a mais de 4 quilômetros de distância de uma sala de vacina. No total, 4.790 municípios apresentaram tendência de agudização de casos de Sindrome Respiratória Aguda Grave. As regiões Norte e Nordeste apresentaramo maior número de Unidades Básicas de Saúde com mais de 5 quilômetros de distância de antenas de celular. O Plano nacional de vacinação requer o uso de estratégias inovadoras para contornar os desafios do país. O uso de metodologias baseadas em inteligência artificial espacial pode contribuir para melhoria do planejamento das ações de resposta à COVID-19.

Palavras-chave Análise espacial, Inteligência artificial, Vacinação em massa, Programas de imunização, Mapeamento geográfico 


\section{Introdução}

A pandemia da COVID-19 atingiu, rapidamente, mais de 188 países e afetou, até o momento, mais de 106 milhões pessoas e causando mais de 2,3 milhões de mortes ${ }^{1}$. A magnitude e a letalidade da pandemia, em um contexto de carência de recursos terapêuticos e preventivos e de sobrecarga dos serviços, multiplicaram a adoção de medidas de distanciamento social e lockdown, com efeitos em toda a economia mundial ${ }^{2,3}$. Após 12 meses de pandemia declarada, ainda não se sabe ao certo os tratamentos que podem ser eficazes a ponto de reduzir a letalidade da doença $a^{4}$.

No início de 2021, com a multiplicação de vacinas com eficácia e segurança comprovadas ${ }^{5}$, o principal desafio associado ao enfrentamento da COVID-19 perpassa a imunização rápida e em massa da população ${ }^{6}$. Até o dia 7 de fevereiro de 2021, 81 países já haviam iniciado suas campanhas de vacinação, atingindo 99 milhões de pessoas? ${ }^{7}$.

As campanhas de saúde funcionam como uma das principais intervenções utilizadas para o controle de uma doença infectocontagiosa ${ }^{8}$. O desafio de se delinear campanhas de saúde efetivas já se fazia presente anteriormente, com as vacinas regularmente disponíveis nos serviços de saúde. Até mesmo em países com sistema de saúde consolidado, as coberturas vacinais raramente alcançam $100 \%$, uma vez que não se consegue localizar a totalidade da população-alvo9,10,11. Fragilidades no direcionamento e no dimensionamento dos esforços das campanhas dificultam a mitigação das consequências deletérias associadas às doenças imunopreveníveis, como é o caso dos surtos de sarampo no Brasil ${ }^{9,10,11}$. A queda na cobertura vacinal brasileira nos últimos anos tem sido associada a diferentes fatores, tais como a diminuição da percepção de risco das doenças, o aumento de crises sociais, políticas e econômicas, o aumento da recusa vacinal, disseminação de crenças e informações falsas sobre vacinas, problemas logísticos, além da forte influência da pandemia na redução da busca por imunização na rede básica do SUS ${ }^{9-11}$.

Dentre os desafios para a estruturação de campanhas de saúde efetivas, a definição e localização da população-alvo surgem como pontos a serem discutidos. Somente a partir da correta identificação do público-alvo é possível estimar os recursos necessários e os custos associados à condução das campanhas em saúde. Estimar a população-alvo de uma campanha é uma tarefa desafiadora, uma vez que a principal fonte de dados para tal ação são os censos populacionais. Para muitos países, não há informações atualizadas originárias de censos para os últimos 15 $\operatorname{anos}^{12}$. No Brasil, a pandemia se sobrepôs à atualização do censo de 2010, impedindo seu curso regular. Assim, é factível estimar que não haverá disponibilidade de dados populacionais primários até meados de 2024, o que nos deixará por quase 15 anos sem um panorama in loco da situação demográfica do país. Em que pese a existência de estimativas intercensitárias realizadas pelo IBGE, o processo de transição demográfica do Brasil tem contribuído para a estagnação de crescimento populacional em algumas localidades e a redução de crescimento em outras, o que pode afetar a precisão das estimativas realizadas entre os censos ${ }^{13,14}$. Sem estimativas fidedignas dos quantitativos populacionais e de sua distribuição espacial, as ações com base em denominadores populacionais ficam comprometidas.

Diante dessa realidade desafiadora, o Plano Nacional de Operacionalização da Vacinação Contra a COVID-19 $9^{15}$, terá dificuldades para a implementação dos eixos avaliativos que requerem informações atualizadas acerca da distribuição da população-alvo e do dimensionamento do esforço necessário para a população elegível. Parte da estratégia para melhor estruturar campanhas de saúde efetivas se baseia na utilização de abordagens de microplanejamento que, por sua vez, pode ser entendido como um conjunto de ações estratégicas, orientadas para o cumprimento dos objetivos das campanhas em saúde ${ }^{16}$. O microplanejamento congrega informações locais, análise de logística, ferramentas de monitoramento rápido e bases de dados populacionais para a criação de um plano de ação destinado a efetivar uma determinada campanha em saúde ${ }^{17}$. Apesar da potencialidade de soluções de microplanejamento, a falta de conhecimento da situação de cada território, a ausência de estimativas da população residente atualizadas e a escassez da geolocalização dos serviços de saúde contribuem para a diminuição de suas potencialidades.

A realização de microplanejamento baseado em estimativas de população desatualizadas ou imprecisas, com distâncias equivocadas entre as comunidades e os pontos de prestação de serviços, pode resultar em uma má alocação de recursos, desperdício de doses de vacina e na impossibilidade de alcance à população alvo da campanha. Dentre as inúmeras inovações voltadas ao aprimoramento de microplanejamento de campanhas de saúde, pode-se destacar a utilização de soluções de geoprocessamento, como 
os Sistemas de Informações Geográficas (SIG), uma alternativa ao processo de desenvolvimento tradicional ${ }^{16}$. As soluções SIG podem ser usadas para identificar comunidades elegíveis, incluindo aquelas não documentadas anteriormente. Dentre as vantagens da utilização desse tipo de abordagem, destaca-se tanto a capacidade de estimar com precisão quantitativos populacionais quanto sua localização com base na análises de imagens de satélite ${ }^{18}$. Dados mais precisos, disponíveis durante a fase de planejamento, permitem uma programação mais eficiente dos recursos necessários para a realização do microplanejamento de campanhas.

O plano nacional menciona sobre a importância de ações de microplanejamento, sem entretanto definir detalhes ${ }^{15}$. Desta forma, torna-se essencial o uso de novas tecnologias para estimar as necessidades dos programas de vacinação e concretizar suas estratégias de cobertura ante à desatualização e incompletude de informações disponíveis. Também se observa a escassa utilização de recursos geográficos para apoiar a localização de grupos vulneráveis e prioritários para imunização, o que torna a inteligência artificial geoespacial peremptória diante dos desafios inerentes à implantação do Plano Nacional de Operacionalização da Vacinação Contra a COVID-19.

Ao considerar os desafios supracitados, bem como os eixos estruturados junto ao plano nacional de vacinação contra COVID-19 e suas lacunas, o presente trabalho demonstra como o uso de inteligência artificial espacial pode ser aplicado para organizar informações, de forma tal que gestores em saúde possam planejar ações eficazes para implementar o plano de imunização contra a COVID-19 no Brasil.

\section{Métodos}

\section{Desenho do estudo e contextualização}

Realizou-se um estudo ecológico transversal em abordagem espaço-temporal, com dados secundários. A unidade de análise foram as Unidades Básicas de Saúde (UBS) existentes no Brasil, em novembro de 2020, registradas junto ao Cadastro Nacional de Estabelecimentos de Saúde (CNES).

\section{Fontes de dados}

Para a realização do presente estudo foram utilizadas seis fontes de dados secundárias (Quadro 1).

\section{Análise de dados}

Para se avaliar de que forma a elaboração de microplanos de vacinação para a COVID-19 pode se beneficiar de soluções de SIG com inteligência artificial (IA) foram estruturadas em quatro passos analíticos: 1) geolocalização das UBS com sala de vacina ativa em novembro de 2020; 2) criação de áreas de abrangência e estimação de população adscrita às salas de vacina ativas; 3) exame da distância entre as UBSs com sala de vacina e torres de celular aptas a apoiar serviços de internet móvel; e 4) análise da tendência espaço-temporal de agudização de casos de COVID-19, por meio de clusterização espaçotemporal de hospitalizações por SRAG vinculadas à COVID-19.

Ao agregar as informações da população dentro da faixa etária alvo do plano, sua localização, a situação epidemiológica da COVID-19, bem como o acesso à internet nas salas de vacina será possível elencar regiões prioritárias no país, de acordo com os critérios definidos no plano de vacinação para COVID-19.

\section{Primeiro passo - geolocalização das UBS do país}

A utilização do CNES para geolocalização das UBS precisou ser conjugada a partir de duas partes. A primeira parte foi extraída a partir do File Transfer Protocol (FTP) ${ }^{19}$ do DATASUS. Essa parte do CNES lista todos os estabelecimentos de saúde ativos e suas instalações físicas. Dessa forma, foi possível extrair do universo de 330.652 estabelecimentos, 47.543 classificados como "Posto de saúde" ou "Centro de saúde / unidade básica”. Desses, 32.226 possuíam pelo menos uma sala de imunização registrada. Apesar do FTP conter o detalhamento das instalações físicas, ele não disponibiliza as coordenadas geográficas de localização das unidades. Essa informação, em sua versão mais atual, está disponibilizada em outro repositório de dados, o OpenData$s u s^{20}$. Esse repositório, apesar de disponibilizar as coordenadas, divulga a informação com uma inconsistência grave, referente ao separador de casas decimais das coordenadas de latitude e longitude. Assim, uma coordenada que deveria ser representada como $-4,4568898$ de latitude 
Quadro 1. Fonte de dados secundários.

\begin{tabular}{|l|l|l|}
\hline \multicolumn{1}{|c|}{ Base de dados } & \multicolumn{1}{|c|}{ Finalidade } & Abrangência espaço temporal \\
\hline $\begin{array}{l}\text { Cadastro nacional de } \\
\text { estabelecimentos de saúde }\end{array}$ & $\begin{array}{l}\text { Obtenção do detalhamento referente às } \\
\text { UBS, no que tange à existência de salas de } \\
\text { vacina, geolocalização e status ativo }\end{array}$ & $\begin{array}{l}\text { Novembro de 2020, todas as } \\
\text { salas existentes no país. }\end{array}$ \\
\hline OpenStreetMap & $\begin{array}{l}\text { Malha de arruamento do Brasil para } \\
\text { a criação de áreas de abrangência que } \\
\text { reflitam as possibilidades de deslocamento } \\
\text { real da população }\end{array}$ & $\begin{array}{l}\text { Abril de 2020, todo o território } \\
\text { nacional. }\end{array}$ \\
\hline WorldPop ${ }^{19}$ & $\begin{array}{l}\text { População dasimétrica para obtenção de } \\
\text { estimativas de quantitativo populacional e } \\
\text { localização que refletissem a situação em } \\
\text { 2020 }\end{array}$ & $\begin{array}{l}\text { Abril de 2020, todo o território } \\
\text { nacional. }\end{array}$ \\
\hline $\begin{array}{l}\text { Estações de rádio base de } \\
\text { serviço móvel pessoal }\end{array}$ & $\begin{array}{l}\text { Obtenção da localização das antenas }{ }^{20} \text { de } \\
\text { celular parâmetros sobre a disponibilidade } \\
\text { de sinal de internet móvel no país (https:// } \\
\text { doi.org/10.6084/m9.figshare.13528424) }\end{array}$ & $\begin{array}{l}\text { Dezembro de 2020, todo o } \\
\text { território nacional. }\end{array}$ \\
\hline SIVEP- gripe & $\begin{array}{l}\text { Casos de síndrome respiratória } \\
\text { aguda grave (SRAG) em decorrência } \\
\text { de COVID-19, ou sem classificação } \\
\text { definida para identificação de padrões de } \\
\text { hospitalização de casos. }\end{array}$ & $\begin{array}{l}\text { Todos os casos registrados } \\
\text { até 07/01/2021, o que } \\
\text { totaliza 941.251 classificados } \\
\text { como COVID-19 ou com } \\
\text { classificação pendente. }\end{array}$ \\
\hline Projeção populacional do IBGE & $\begin{array}{l}\text { Cálculo do percentual de idosos } \\
\text { identificados através da abordagem de } \\
\text { inteligência artificial espacial. }\end{array}$ & $\begin{array}{l}\text { Estimativa para 2019, por faixa } \\
\text { etária }{ }^{21} .\end{array}$ \\
\hline
\end{tabular}

Fonte: Elaborado pelos autores.

sul, é apresentada como 44.568.898. Esta forma de apresentação do arquivo disponibilizado pelo OpenDatasus impede a utilização dos dados mais atuais de localização. Para resolver esse ponto, os dados das coordenadas foram processados para a correção do erro de separação de casas decimais. Para cada estado brasileiro foram extraídos os limites geográficos referentes aos seus quatro extremos espaciais. Dessa forma, as coordenadas mais ao norte e sul, bem como aquelas mais ao leste e oeste, foram utilizadas como limites de validação para as coordenadas brutas obtidas junto ao OpenDatasus. Ao se validar as coordenadas brutas com problema e corrigi-las, foi possível reconstruir a informação permitindo a geolocalização de 31.727 das 32.226 UBS com sala de vacinação. Dada a complexidade de processamento dessa informação em função da necessidade de validação espacial e buscando garantir a replicabilidade de nossos achados a base de dados foi disponibilizada no repositório Figshare em: https://doi.org/10.6084/m9.figshare.13528421.v1

\section{Segundo passo - definição das áreas de abrangência de cada UBS}

Em seguida, as UBS foram utilizadas como base para a criação de áreas de abrangência. A Política Nacional de Atenção Básica (PNAB) de $2017^{21}$ estipula que a prestação dos serviços de atenção primária devem ser prestados o mais próximo possível de sua população adscrita. Assim se espera que as UBS sejam responsáveis pela prestação de serviços à população adjacente. Infelizmente, o Brasil não dispõe de um repositório centralizado que congregue as representações espaciais das áreas de abrangência das $\mathrm{UBS}^{22}$. Como não existe um repositório digital, foram criadas áreas de abrangência para as UBS por meio de polígonos de isodistância. Esses polígonos delimitam uma área, dentro da qual se pode acessar a UBS, com uma distância máxima de até 4 quilômetros de deslocamento, pois tal distanciamento foi escolhido como proxy de um deslocamento de aproximadamente 1 hora à pé. Qualquer necessidade de vencer distâncias superiores a essa foge ao que está preconizado pela PNAB, por se configurar como uma barreira de 
acesso geográfico. Os polígonos de isodistância foram criados analisando-se a malha de arruamento real de todo o Brasil para 2020, obtida junto ao OpenStreetMap ${ }^{23}$. Ao todo foram analisadas cerca de 10 milhões de ruas. Fontes de transporte por trem e barco também foram consideradas, especialmente na Região Norte. Cada polígono resultante foi estruturado para não se sobrepor a outros polígonos da análise, o que maximizou a extensão territorial coberta pelas UBS.

\section{Terceiro passo - atribuição de população adscrita às áreas de abrangência com base em estimações populacionais utilizando inteligência artificial geoespacial}

O terceiro passo consistiu na utilização dos polígonos gerados no passo 2, para cada unidade de saúde, como área de abrangência para a sumarização da população ali inserida.

A população dasimétrica é o nome atribuído às estimativas populacionais obtidas por meio da análise de dados de imagens de satélite e de covariáveis espaciais, por meio de algoritmos de inteligência artificial ${ }^{24}$. Os dados utilizados para tal análise são coletados por meio de sensores incorporados em satélites. Esses sensores são capazes de captar, por exemplo, presença de luzes noturnas e refração de luz solar por construções artificiais $^{24}$. A partir da análise desses dados é possível criar projeções populacionais que levam em conta as taxas de variação populacional distribuídas por área de construção artificial. Dados de censos anteriores conjugados com a análise de variação de cobertura de solo, os algoritmos de IA, são capazes de estimar a quantidade de pessoas por área coberta com construções artificiais identificadas pelas imagens de satélite. A granularidade de predição é de 1 quilômetro quadrado.

Há diversas fontes de população dasimétrica e a opção do presente trabalho foi por utilizar aquela gerada pelo WorldPop ${ }^{24}$, pois oferece a possibilidade de estratificação das estimativas por faixas etárias. Além disso, as análises de precisão entre os valores das estimativas populacionais locais e aqueles obtidos pela WorldPop foram aqueles que apresentaram as menores discrepâncias, graças à utilização de abordagens baseadas em inteligência $\operatorname{artificial}^{25}$.

Dessa forma, o arquivo raster com a distribuição de população dasimétrica, obtida junto ao WorldPop ${ }^{24}$, foi analisado por meio da abordagem de estatística zonal, do software ArcGis Pro. Esta abordagem soma os valores dos pixels correspondentes aos quantitativos de população por quilômetro quadrado, e atribui o valor resultante a uma área de abrangência de uma UBS com sala de vacinação ativa.

O resultado obtido nesse passo é a estimativa de população, referente a abril de 2020, para os grupos etários selecionados, para cada sala de vacina ativa no país. Com base neste dado, passa a ser possível o desenho de ações estratégicas para a formalização de microplanos customizados para a cobertura de bolsões de população prioritária, bem como a identificação de áreas descobertas por serviços de saúde, que exigirão ações de vacinação volante.

\section{Quarto passo - análise de priorização de grupos estratégicos, conforme diretrizes do plano de vacinação para COVID-19}

O plano de vacinação lista três eixos de monitoramento da campanha de imunização: Avaliação e identificação da estrutura existente na rede; Processos; Indicadores de intervenção ${ }^{15}$. O primeiro eixo perpassa os dados que serão utilizados para a construção dos indicadores de monitoramento subsequente do plano. Os dois últimos eixos compreendem as ações que serão empreendidas somente após o início da vacinação. Dentro desses eixos faz-se necessário o monitoramento das salas de vacina existentes, das internações por Síndrome Respiratória Aguda Grave (SRAG), definição população alvo a ser vacinada, necessidade de equipes para vacinação volante, existência de internet nas salas de vacina para registro eletrônico das ações de vacinação, bem como o dimensionamento de insumos para a implementação do plano. Assim, o quarto passo analítico desse manuscrito caracteriza o território brasileiro em termos dos indicadores de monitoramento do eixo 1 do plano. A população alvo prioritária, acima de 60 anos, foi identificada e vinculada a uma sala de vacinação nos passos analíticos de 1 a 3 . No quarto passo foi analisada a proximidade das salas de vacina para com antenas de celular capazes de suportar tráfego de internet para registro de doses e, por último, a análise da tendência espaço-temporal de casos de SRAG, para indicar no espaço aquele conjunto de municípios que vem apresentando um incremento de casos graves de COVID-19. A análise de tendência espaço-temporal foi realizada por meio da técnica de space time cube, com clusterização por emerging hotspot analysis ${ }^{26}$, e que utiliza como variável de desfecho a incidência de SRAG por 100 mil habitantes associada à COVID-19. As análises referentes ao passo 1 foram realizadas com o software estatístico $\mathrm{R}$ e com os pacotes WOPR ${ }^{27}$. Os passos subsequentes foram realizados com o ArcGis Pro 2.5. 


\section{Resultados}

Os primeiros três passos buscaram identificar as UBS com sala de vacinas, bem como a população maior de 60 anos vivendo em até quilômetros de distância a pé de cada estabelecimento. A Tabela 1 sumariza o resultado dos três primeiros passos analíticos. Todos os arquivos com as bases de dados utilizadas podem ser encontradas nesse repositório do FigShare, cujo link é (https://doi. org/10.6084/m9.figshare.13528577.v1). A Figura 1 demonstra como se representam as áreas de abrangência construídas com a abordagem metodológica proposta neste manuscrito. As linhas pontilhadas demarcam as áreas de abrangências das UBS em Belém, Pará (Figura 1A). A seção 1B demonstra a distribuição da população dasimétrica para mesma região, em que as áreas com cores escuras representam regiões com maior quantidade de pessoas.

O Brasil conta com 32.474 salas de vacinas, distribuídas em 32.226 UBS (Tabela 1). A Região Nordeste apresentou a maior quantidade de salas com 13.203 instalações dessa natureza. Das 32.226 UBS foi possível obter as coordenadas geográficas de 31.727. Ao se aplicar a solução para a construção de áreas de abrangência em cada UBS foi possível obter 23.171 áreas de abrangência. $O$ número de áreas inferior ao total de UBS se deve a dois fatos: parte das unidades não foi possível obter uma ligação com a malha de arruamento próxima, por estarem localizadas em zonas não mapeadas pelo OpenStreetMap, o que totaliza 2.308 UBS nessa situação. As demais não receberam uma área de abrangência por estarem próximas a outra UBS e terem sido abarcadas pela área de abrangência de outra unidade. Assim, das 31.727 UBS geolocalizadas foram analisados dados de população dasimétrica referentes a 29.419 UBS.

$\mathrm{Na}$ análise de $92,72 \%$ das UBS geolocalizadas do país foi possível identificar 23.792.907 pessoas acima de 60 anos em até 4 quilômetros de distância das UBS consideradas, que representam $82 \%$ da população idosa do país. Para os $18 \%$ restantes será necessário um deslocamento maior que 4 quilômetros a pé para acessar uma UBS com potencial de vacinar contra a COVID-19. Na Região Sul, $24 \%$ dos idosos viviam a mais de 4 quilômetros de distância de uma UBS com sala de vacina. O contexto sociodemográfico com uma grande proporção de pessoas idosas enfrentando potenciais barreiras de acesso à imunização representará um desafio adicional às estratégias de vacinação.
No que se refere à proximidade com antenas de celular, exposto no link (https://doi. org/10.6084/m9.figshare.13528424.v1), 27,388 UBS com sala de vacina estão a até 2,5 quilômetros de distância linear de uma torre de telefonia celular. Desprezada a existência de obstáculos geográficos tais como vales e montanhas, se espera que essas unidades tenham a potencialidade de acessar redes de dados móveis para a transmissão de informações relacionadas ao registro da vacinação. As Regiões Norte e Nordeste foram as que apresentaram o maior quantitativo de UBS a mais de 5 quilômetros de distância de estações de rádio base de celular. Para as unidades dessts localidades será mais difícil o registro eletrônico de doses e, assim, de monitorar o andamento da campanha de vacinação.

A Figura 2 apresenta os clusters espaço-temporais referentes à incidência de SRAG para o ano de 2020 no país. As cores quentes apresentam uma tendência de incremento da variável analisada ao longo do tempo. O detalhamento completo das categorias pode ser encontrada no website da ESRI ${ }^{28}$. A situação do Brasil, no que tange à incidência de SRAG em 2020, foi de incremento ao longo das 53 semanas epidemiológicas, a considerar a dominância de cores do espectro quente no mapa.

As regiões categorizadas como new hot spots são regiões que nunca foram classificadas como zonas quentes e que nos momentos finais da observação temporal passaram a ser classificadas como tal. Nestes lugares, a incidência de SRAG em decorrência da COVID-19 sofreu incremento ao final de 2020, o que sugere um potencial significativo para agudização de pacientes. Os três estados da Região Sul foram categorizados como Oscillating hot spot, o que significa que eram áreas de cold spot de internações por SRAG no passado, mas que se converteram em hot spots nos pontos mais recentes da escala temporal analisada. Essa situação atingiu os estados de Minas Gerais, Goiás, Tocantins, Rondônia, Acre, leste do Pará, o litoral do Nordeste e Mato Grosso. Por fim, os estados do Amazonas, Mato Grosso do Sul, Sul de Roraima e Amapá foram avaliados como Sporadic hotspot que define um agrupamento de municípios que alternam uma situação de clusterização não significante, com classificação de hotspot. Cabe destacar a situação do sudoeste de São Paulo, que apresentou um cluster de Intensifying hotspot que marca a situação das cidades com classificação de hot spot em $90 \%$ do tempo, e que nos últimos pontos da escala temporal a significância estatística do agrupamento se tornou mais intensa. 


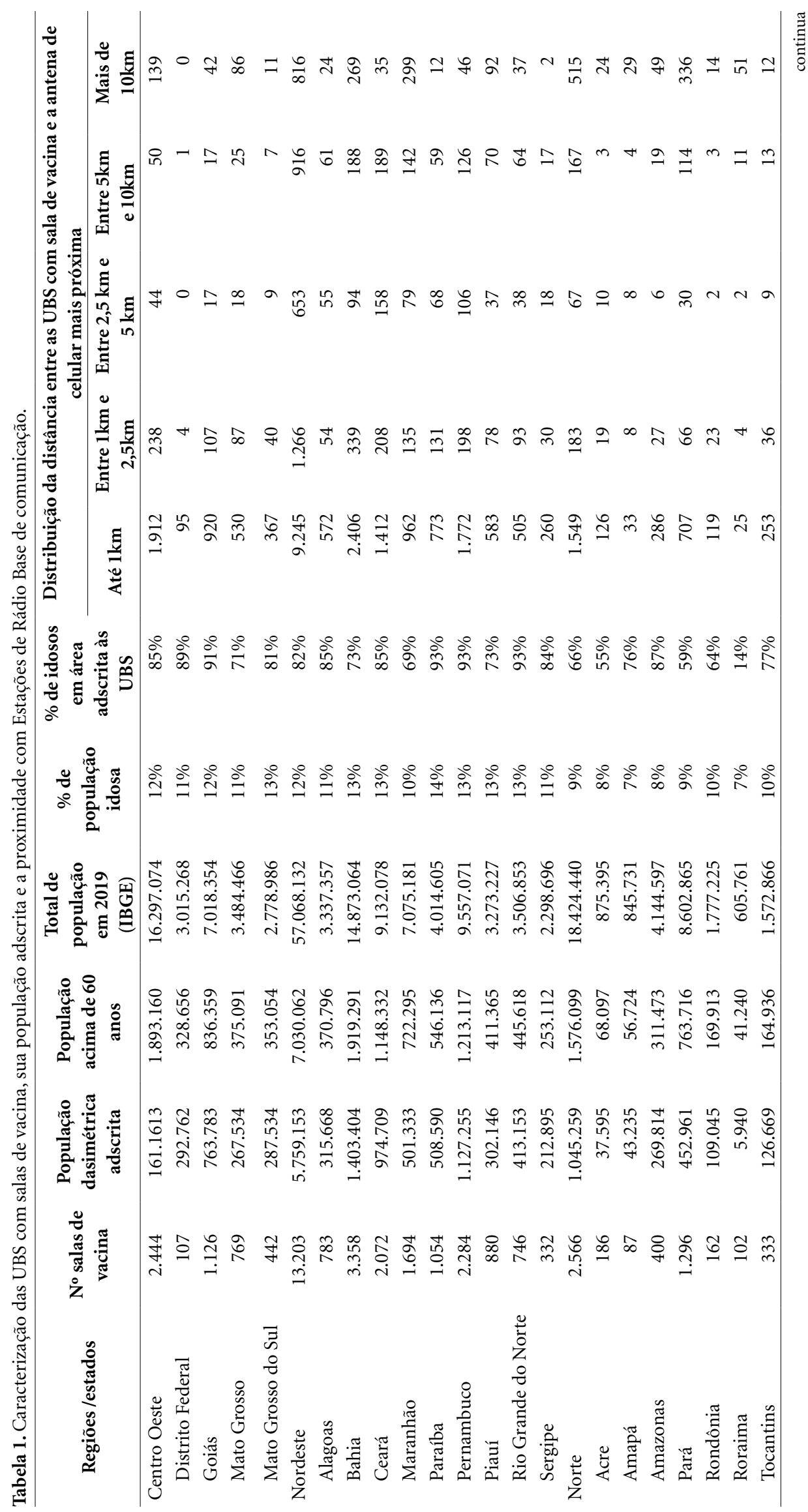




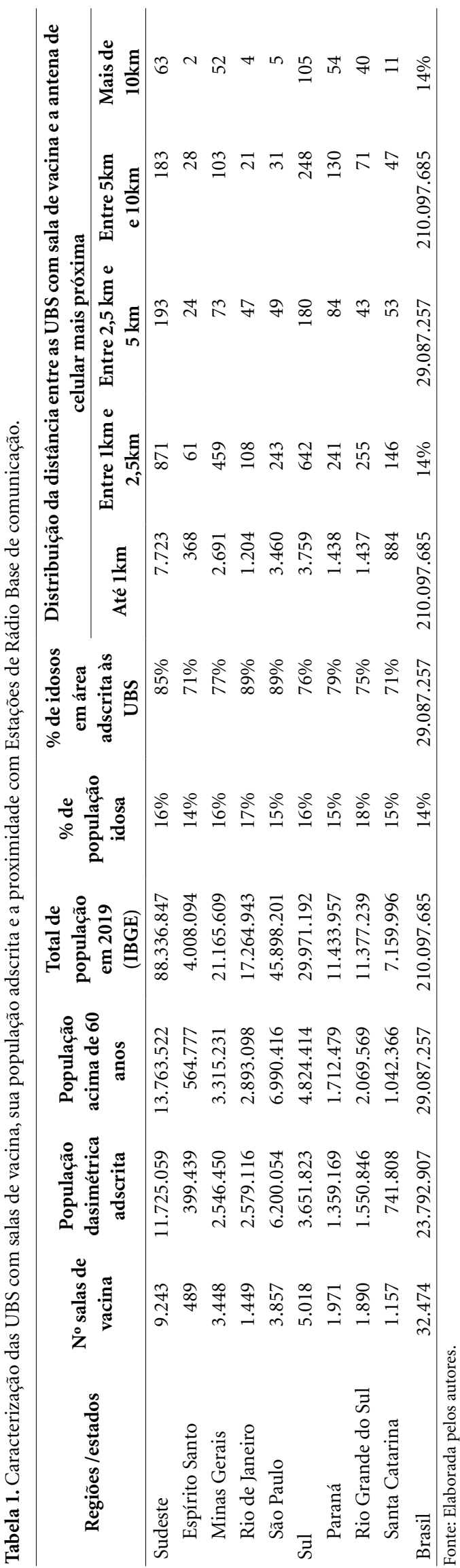

A Tabela 2 detalha a distribuição da clusterização espaço temporal de SRAG para $5.561 \mathrm{mu}-$ nicípios brasileiros. Nove municípios foram excluídos da análise por inconsistências nos dados. Do total de municípios analisados 3.253 foram considerados como Oscillating hotspot. Sendo que 1.102 estão na região Sul. A Região Nordeste apresentou 518 municípios categorizados como New Hot Spot, assim 28\% de suas cidades apresentaram um padrão de incremento de SRAG no final do ano de 2020.

\section{Discussão}

O objetivo do presente manuscrito foi analisar de que forma o uso de inteligência artificial espacial poderia fornecer informações para que gestores em saúde possam planejar ações eficazes para implementar o plano de imunização contra a COVID-19 no Brasil.

O fomento a ações para a mitigação da pandemia no Brasil faz-se peremptória em função da magnitude de seu impacto sobre a nossa sociedade. Até 7 de Fevereiro, a COVID-19 foi responsável por 231 mil óbitos no país ${ }^{29}$. Estudos demográficos estimam um excesso de mortalidade até a semana epidemiológica 52 de 2020 a cerca de 234 mil óbitos $^{30,31}$. Esse dado sugere que até 34 mil mortes adicionais podem ser atribuídas à COVID-19 após os procedimentos de investigação de óbito, apenas em 2020.

As consequências nefastas vinculadas à COVID-19 têm impingido aos governantes a busca de soluções para a retomada da atividade econômica e restabelecimento da normalidade cotidiana. Apesar desses anseios perfazerem o imaginário social da população, não há atalho capaz de superar os desafios que foram colocados pela COVID-19. A necessidade de gerar respostas fez com que o Ministério da Saúde do Brasil elaborasse o Plano nacional de vacinação contra a COVID-19. Este foi alvo de críticas pela comunidade acadêmica em função do diminuto grau de detalhamento fornecido para definir estratégias de operacionalização.

Com base nesta lacuna, o presente manuscrito visou apresentar estratégias, amparadas em geoprocessamento e inteligência artificial, que podem ser utilizadas para fomentar ações pragmáticas de priorização da população mais vulnerável. O uso de sensoriamento remoto, imagens de satélite e algoritmos de inteligência artificial para projeção de população surge como uma via possível para se identificar bolsões de população 


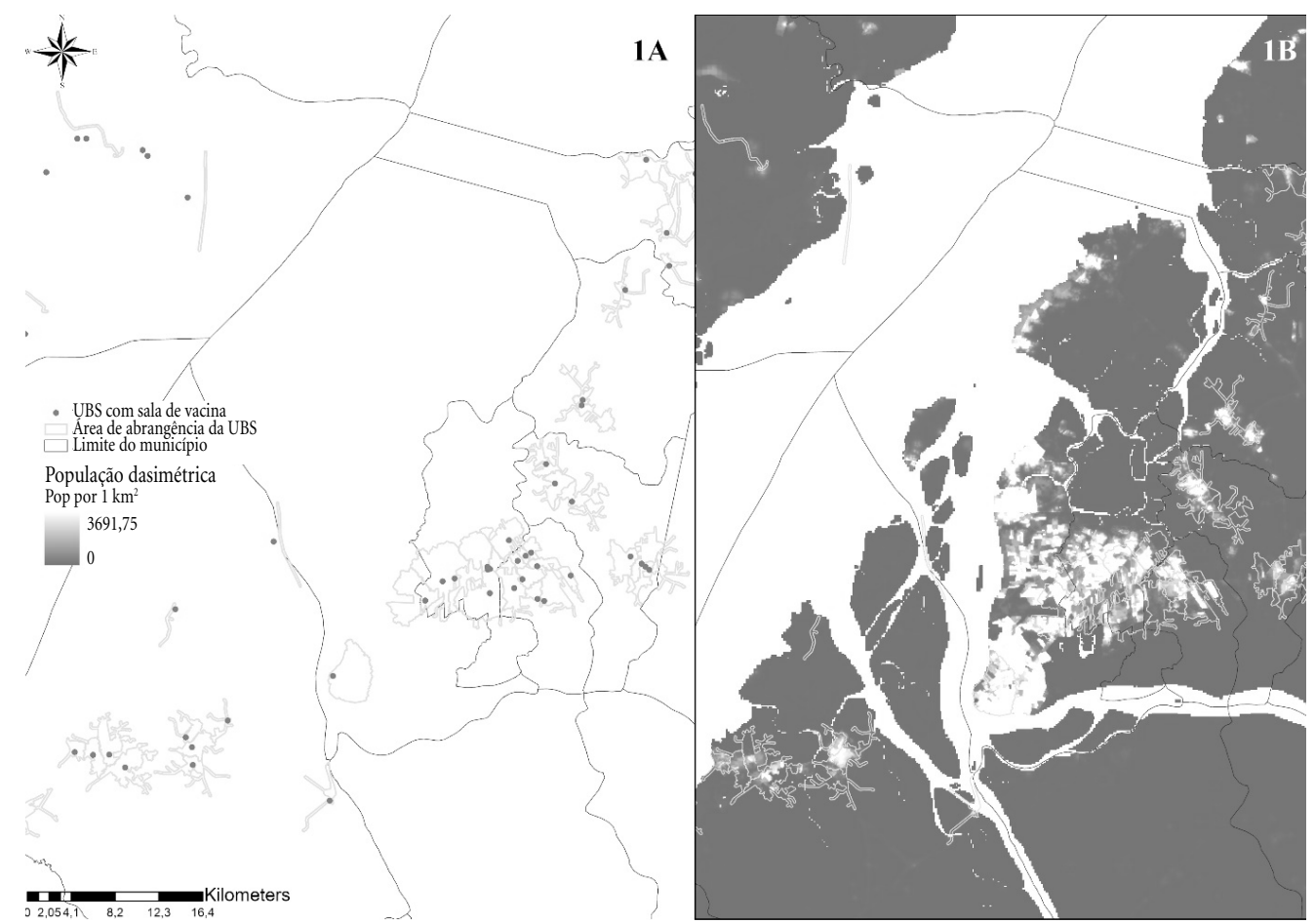

Figura 1. Representação das áreas de abrangência criadas e distribuição da população dasimétrica para Belém do Pará, 2020.

Fonte: elaborado pelos autores.

elegível, áreas com dificuldade de acesso passíveis de abordagens de vacinação extramuros, bem como a estimação de recursos para a condução de campanhas em regiões com surto ativo de doenças.

Em regiões urbanas o limite testado de 4 quilômetros de distância a pé pode ser vencido com ajuda de transporte público, mas em zonas rurais ou remotas uma distância superior a essa pode se configurar como uma barreira de acesso. A utilização desse limite de proxy é alternativa valiosa frente a inexistência de um repositório digital nacional, que contemplasse as áreas de abrangência reais das UBS do país ${ }^{32}$. Algum esforço já foi conduzido para se tentar superar essa lacuna de representação das áreas de adscrição de população das UBS brasileiras ${ }^{22}$, mas ainda resta um longo caminho até a solução definitiva. Dada a urgência de se propor soluções para catalisar as possibilidades de imunização da população, a abordagem detalhada aqui é a que mais se aproxima de forma propositiva para a superação desses desafios.

Ao analisar a tendência espaço temporal de SRAG ao longo de 2020, a população dasimétrica adscrita às UBS, o percentual de população idosa nos municípios e existência de redes de comunicação com suporte à internet móvel é possível vislumbrar condições mais concretas para a abordagem da população prioritária definida junto ao Plano Nacional para vacinação contra a COVID-19.

As bases de dados divulgadas conjuntamente com esse manuscrito permitem que os gestores identifiquem as UBS das quais são responsáveis, obtenham uma estimativa da população elegível próxima à unidade, avaliem a disponibilidade ou não de internet em cada sala de vacina $e$ confrontem essas informações com a tendência de agudização de casos em cada município. Da- 


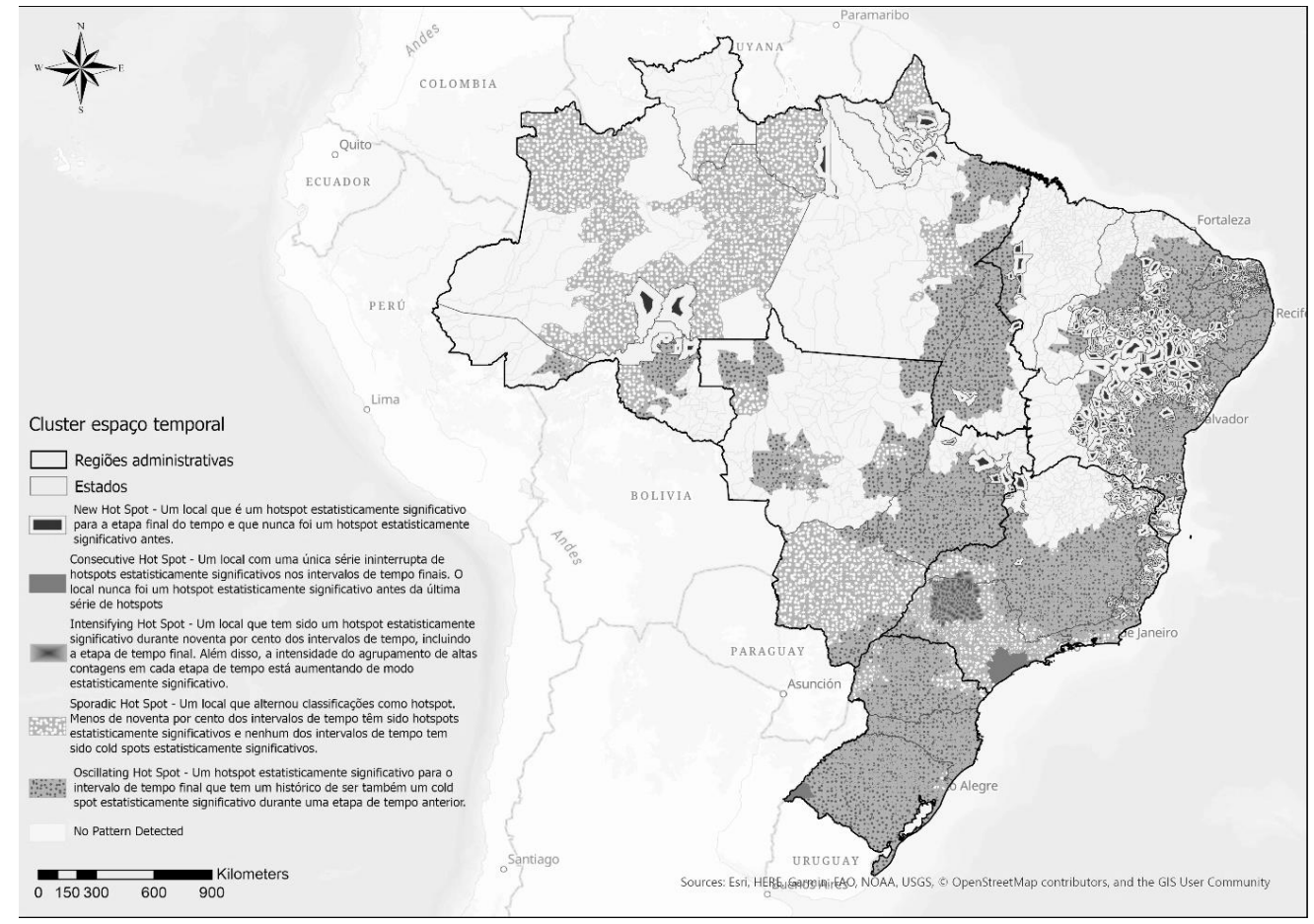

Figura 2. Clusters espaço-temporais referentes à incidência de SRAG para o ano de 2020.

Fonte: elaborado pelos autores.

dos do repositório Figshare, nos links https://doi. org/10.6084/m9.figshare.13528577.v1 e https:// doi.org/10.6084/m9.figshare.13528430.v1, serviram para dar embasamento à pesquisa. Assim, gestores municipais podem ter acesso a informações para implementação de microplanos de vacinação, identificando quantitativos populacionais e áreas remotas, com população em situação de potencial desassistência. Gestores estaduais podem ter a noção macro de seu estado e eleger áreas prioritárias para distribuição de doses de vacina, com o objetivo de minimizar casos graves e o esgotamento de leitos de UTI.

Os desafios regionais do Brasil são diferenciados. O Plano prevê que o registro das doses aplicadas será vinculado à Rede Nacional de Dados em Saúde (RNDS) ${ }^{33}$ e, portanto, ao Conecte SUS. Ao considerar a proximidade de serviços de internet, pode-se vislumbrar que o registro eletrônico de dados será um desafio nas regiões Norte e Nordeste. Assim, a proposta de um registro eletrônico de doses precisará de ajustes para comportar parte da coleta de dados de modo offline. Além disso, dadas as limitações de conectividade, o seguimento de eventos adversos e associados ao processo de vacinação não poderá ser online. Isso pode fazer com que o monitoramento de problemas vinculados à campanha de vacinação seja postergado, o que impedirá a adoção de medidas protetoras de forma eficaz. Com os nossos resultados é possível ter um panorama sala a sala, em relação à potencial conectividade com redes móveis de comunicação.

Aproximadamente, 5,2 milhões de pessoas estão a mais de 4 quilômetros das UBS, o que pode exigir estratégias de vacinação volante. Na região Sul, onde $24 \%$ dos idosos estão nesta condição, observou-se a tendência de incremento espaçotemporal de casos de SRAG em 93\% $(n=1.102)$ dos 1.189 municípios. Assim, é recomendável a adoção de estratégias de busca ativa de pessoas mais intensa, dada as características das unidades e o perfil epidemiológico da doença na região. Para 4.790 municípios brasileiros há diferentes padrões de incremento dos casos de SRAG nas últimas semanas epidemiológicas do ano de 2020. Essa situação coloca extensas porções de todas as regiões do país em uma situação delicada. 


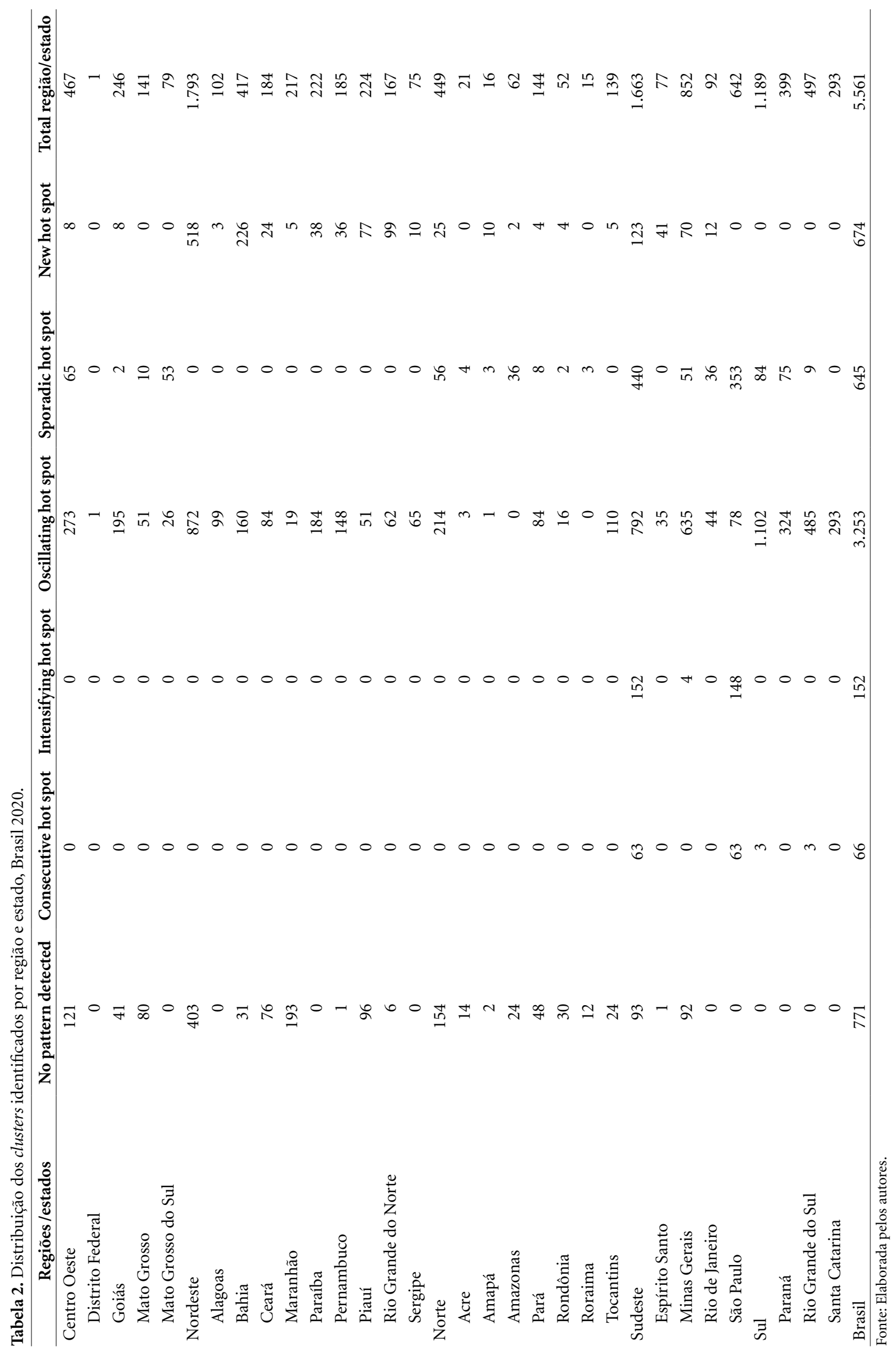


Parte da dificuldade em estruturar campanhas de vacinação efetivas está associada à qualidade das estratégias de microplanejamento elaboradas pelos municípios, conforme a tradição do SUS. Usualmente, o Ministério da Saúde define as diretrizes da campanha, se responsabiliza pelas ações de financiamento, comunicação, suprimento, o que inclui os imunobiológicos e logística, enquanto a execução das ações de vacinação fica a cargo dos municípios. O Brasil é um país heterogêneo com múltiplas realidades que afetam as capacidades referentes a planejamento, ao uso de ferramentas de análise de dados e ao planejamento de recursos logísticos e humanos. Alguns municípios irão alcançar efetivamente os resultados almejados pela campanha, ao passo que outros terão dificuldades. Ao final do processo, o Ministério da Saúde é o responsável pelas coberturas vacinais. A forma de organização atual de campanhas tem como elo fraco exatamente a ferramenta mais poderosa para a consecução dos resultados preconizados: as ações de microplanejamento. Essa forma de conduzir campanhas em saúde vem apresentando problemas ao longo dos últimos cinco anos tais como redução de coberturas ${ }^{34}$, e surtos de doenças imunopreveníveis ${ }^{35-37}$. Esse cenário aumenta as dificuldades da campanha de COVID-19 para além da escassa disponibilidade de vacinas e insumos.

O presente manuscrito combinou estratégias analíticas inovadoras para superar alguns dos desafios associados à estruturação da campanha de vacinação contra a COVID-19, com vistas a gerar evidências sobre os indicadores no plano nacional de vacinação. Assim, todos os dados utilizados para a geração das análises supracitadas e seus respectivos resultados estão disponíveis como material suplementar a esse manuscrito.

Dentre as limitações do estudo, destaca-se a impossibilidade de realizar as estimações com base nas áreas adscritas reais das UBS, além de eventuais divergências entre as populações reais e estimadas através dos dados de satélite, bem como imprecisões nas coordenadas geográficas das UBS disponibilizadas pelo DATASUS. Considerando- se o trade-off entre utilizar dados censitários de 10 anos atrás versus a estimação dasimétrica do ano de 2020, esta última conta com a vantagem de examinar todo o território nacional, identificando assentamentos humanos em áreas que não poderiam ser facilmente identificadas sem o uso de dados de satélite. Além disso, as estimativas do WorldPop foram ajustadas para refletir as estimativas nacionais. Apesar disso, as estimativas estão sujeitas a imprecisões. Por último, para superar as limitações vinculadas às coordenadas do DATASUS, foram adotados passos de validação espacial, tais como a checagem da coordenada, confrontando-se os limites espaciais dos estados. Mas ainda assim, melhorias, por parte do DATASUS, podem ser realizadas no processo de divulgação das coordenadas de unidades de saúde. Uma importante estratégia nesse sentido, infelizmente extinta, foi o Programa de Melhoria do Acesso e da Qualidade, que visitava a cada dois anos todas as UBS do país e registrava suas coordenadas geográficas in loco com o uso de GPS ${ }^{38}$.

Quanto às possibilidades de estudos e ações políticas futuras, cabe destacar dois pontos. $\mathrm{O}$ primeiro refere-se à criação de um repositório nacional com as áreas de abrangências das UBS, iniciativa essencial para viabilizar a qualificação do processo de análises de informações associadas aos serviços de atenção primária no país. Atualmente, há ferramentas de mapeamento colaborativo gratuitas que poderiam servir de suporte para o processo coordenado de geração desse tipo de informação. O segundo ponto diz respeito à necessidade de discutir em profundidade estratégias e ferramentas baseadas em geoprocessamento para o fomento à elaboração de microplanos de campanha de saúde. O uso de tecnologias baseadas em geoprocessamento tem potencial de aumentar a relação custo-efetividade e a eficácia do microplanejamento da campanha de vacinação da COVID-1916,39. A estratégia proposta aqui também fortalece a equidade e a universalidade das campanhas de saúde ao permitir a identificação de forma precisa das regiões críticas para intervenção. 


\section{Colaboradores}

TAH Rocha, GM Boitrago, RB Mônica, DM Silva, DG Almeida, NC Silva, JRN Vissoci, SH Terabe, LA Facchini e C Staton participaram da concepção e elaboração do manuscrito, revisão bibliográfica, análise e discussão dos elementos textuais, revisão crítica. Todos os autores aprovaram a versão final do manuscrito e declaram serem responsáveis por todos os aspectos do trabalho, garantindo sua precisão e integridade.

\section{Referências}

1. World Health Organization (WHO). Coronavirus Disease (COVID-19) Dashboard [internet]. Geneva: WHO; 2021.

2. Flaxman S, Mishra S, Gandy A, Uniwn HJT, Mellan TA, Coupland H, Whittaker C, Zhu H, Berah T, Eaton JW, Monod M. Estimating the effects of non-pharmaceutical interventions on COVID-19 in Europe. Nature 2020; 584:257-261.

3. Leung $\mathrm{K}, \mathrm{Wu}$ JT, Liu D, Leung GM. First-wave COVID-19 transmissibility and severity in China outside Hubei after control measures, and second-wave scenario planning: a modelling impact assessment. Lancet 2020; 395(10233):1382-1393.

4. Pascarella G, Strumia A, Piliego C, Bruno F, Del Buono R, Costa F, Scarlata S, Agró FE. COVID-19 diagnosis and management: a comprehensive review. J Intern Med 2020; 288(2):192-206.

5. Thanh Le T, Andreadakis Z, Kumar A, et al. The COVID-19 vaccine development landscape. Nat Rev Drug Discov 2020; 19(5):305-306.

6. Schaffer Deroo S, Pudalov NJ, Fu LY. Planning for a COVID-19 Vaccination Program. JAMA - J Am Med Assoc 2020; 323(24):2458-2459.

7. World in data. Coronavirus (COVID-19) Vaccinations - Statistics and Research [internet]. Oxford: Our World in Data; 2021.

8. World Health Organization (WHO). Best practices in microplanning for polio eradication. Geneva: WHO; $2021.56 \mathrm{p}$.

9. Cruz A. A Queda da imunização no Brasil. Consensus 2017; 25: 20-29.

10. Sato APS. Pandemic and vaccine coverage: challenges of returning to schools. Rev Saude Publ 2020; 54:115.

11. Portnoy A, Jit M, Helleringer S, Verguet S. Impact of measles supplementary immunization activities on reaching children missed by routine programs. Vaccine 2018; 36(1):170-178.

12. Wardrop NA, Jochem WC, Bird TJ, Chamberlain HR, Clarke D, Kerr D, Bengtsson L, Juran S, Seaman V, Tatem AJ. Spatially disaggregated population estimates in the absence of national population and housing census data. Proc Natl Acad Sci USA 2018; 115(14):3529-3537.

13. Duarte EC, Barreto SM. Transição demográfica e epidemiológica: a epidemiologia e serviços de saúde revisita e atualiza o tema. Epidemiol e Serv Saude 2012; 21(4):529-532.

14. Saad PM. Envelhecimento Populacional: Demandas e possibilidades na área de saúde. Assoc Brasil Estud Populacionais ABEP 2016; 3:153-166.

15. Ministério da Saúde (MS). Plano nacional de operacionalização da vacinação contra a COVID-19. Brasília: MS; 2021.

16. Ali D, Levin A, Abdulkarim M, Tijjani U, Ahmed B, Namalam F, Oyewole F, Dougherty L. A cost-effectiveness analysis of traditional and geographic information system-supported microplanning approaches for routine immunization program management in northern Nigeria. Vaccine 2020; 38 (6):1408-1415.

17. King $\mathrm{MH}$, Martodipoero S. Health microplanning in the developing countries: A systems approach to appropriate technology. Int J Heal Serv 1978; 8(4): 653-664. 
18. Gali E, Mkanda P, Banda R, Korir C, Bawa S, Warigon C, Abdullahi S, Abba B, Isiaka A, Yahualashet YG, Touray K, Chevez A, Tegegne SG, Nsubuga P, Etsano A, Shuaib F, Vaz RG. Revised Household-Based Microplanning in Polio Supplemental Immunization Activities in Kano State, Nigeria. 2013-2014. J Infect Dis 2016; 213(suppl 3):S73-S78.

19. Brasil. Ministério da Saúde (MS). CNES. [acessado 2021 Fev 15]. Disponível em: http://cnes.datasus.gov. br/

20. Ministério da Saúde (MS). Cadastro Nacional de Estabelecimentos de Saúde (CNES). Conjuntos de dados - Open Data; 2020.

21. Brasil. Portaria $\mathrm{n}^{\circ} 2.436$, de 21 de setembro de 2017. Aprova a Política Nacional de Atenção Básica, estabelecendo a revisão de diretrizes para a organização da Atenção Básica, no âmbito do Sistema Único de Saúde (SUS). Brasília: MS; 2017.

22. Rocha TAH, de Almeida DG, do Amaral PVM, Silva NC. Proposta de metodologia para estimar a área de cobertura potencial por equipes de atenção primária. Rev Panam Salud Publica 2019; 43(1):e47.

23. OpenStreetMap. OpenStreetMap. [Software] [acessado $2021 \mathrm{Fev}$ 15]. Disponível em: https://www.openstreetmap.org/copyright?locale $=\mathrm{pt}-\mathrm{BR}$

24. Stevens FR, Gaughan AE, Linard C, Tatem AJ. Disaggregating census data for population mapping using random forests with remotely-sensed and ancillary data. PLoS One 2015; 10(2):e0107042.

25. POPGRID. Leaving no one off the map: A guide for gridded population data for sustainable development. New York: POPGRID; 2020. 7p.

26. Silva NC, Rocha TAH, Amaral PV, Elahi C, Thumé E, Thomaz EBAF, Queiroz RCS, Vissoci JRN, Staton C, Facchini LA. Comprehending the lack of access to maternal and neonatal emergency care: Designing solutions based on a space-time approach. PLoS One 2020; 15(7):e0235954.

27. Leasure D, WorldPop. Wopr: An R package to query the worldPop open population repository, version 0.1.0 [internet]. [cited $2021 \mathrm{Feb} 15$ ] University of Southampton; 2020. Available from: https://eprints.soton.ac.uk/439567/.

28. ESRI. How Emerging Hot Spot Analysis works. Documentation [internet]. ArqGIS Pro 2.7. [cited $2021 \mathrm{Feb}$ 15]. Available from: https://pro.arcgis.com/en/proapp/latest/tool-reference/space-time-pattern-mining/ learnmoreemerging.htm

29. Brasil. Ministério da Saúde (MS). Painel Coronavírus. [acessado 2021 fev 15]. Disponível em: http://www. coronavirus.df.gov.br/

30. Conselho Nacional de Secretários de Saúde. Painel de análise do excesso de mortalidade por causas naturais no Brasil em 2020 [internet]. [acessado $2021 \mathrm{Fev} 14$ ]. Acessado em: https://www.conass.org.br/indicadoresde-obitos-por-causas-naturais/
31. Marinho F, Torrens A, Teixeira R, França E, Nogales AM, Xavier D, Fujiwara T. Aumento das mortes no Brasil, regiões, estados e capitais em tempo de COVID-19: excesso de óbitos por causas naturais que não deveria ter acontecido [nota técnica]. Nova Delhi: Vital Strategies; 2020.

32. Andrade MV, Noronha K, Queiroz Barbosa, Rocha TAH, Slva NC, Calazans JA, Souza MN, Carvalho LR, Souza A. A equidade na cobertura da Estratégia Saúde da Família em Minas Gerais, Brasil. Cad Saude Publica 2015; 31(6):1175-1187.

33. Brasil. Ministério da Saúde (MS). Rede Nacional de Dados em Saúde [acessado 2021 fev 14]. Disponível em: https://rnds.saude.gov.br

34. Arroyo LH, Ramos ACV, Yamamura M, Weiller TH, Crispim JA, Cartagena-Ramos D, Fuentealba-Torres M, Santos DT, Palha PF, Arcêncio RA. Areas with declining vaccination coverage for BCG, poliomyelitis, and MMR in Brazil (2006-2016): Maps of regional heterogeneity. Cad Saude Publica 2020; 36(4):e00015619.

35. Branco VGC, Morgado FEF. O surto de sarampo e a situação vacinal no Brasil. Rev Med Família e Saude Ment 2019; 1(1):74-88.

36. Cavalcante KRLJ, Tauil PL. Risco de reintrodução da febre amarela urbana no Brasil. Epidemiol Serv Saude 2017; 26(3):617-620.

37. Silva Leite FSL, Ramalho MIL, Sousa MNA. Evolução do sarampo no estado de Roraima e a atual situação vacinal no Brasil. Rev Ciência e Desenvolv 2019; 12(1):129-140.

38. Rocha TAH, Silva NC, Thomaz EBAF, Queiroz RCS, Souza MR, Lein A, Rocha JVM, Alvares V, Almeida DG, Barbosa ACQ, Thumé E, Staton C, Vissoci JRN, Facchini LA. Primary health care and cervical cancer mortality rates in Brazil: A longitudinal ecological study. J Ambul Care Manage 2017; 40(Supl. 2):S24-S34.

39. Dougherty L, Abdulkarim M, Mikailu F, Tijani U, Owolabi K, Gilroy K, Naiya A, Abdullahi A, Bodinga H, Olayinka F, Moise I. From paper maps to digital maps: Enhancing routine immunisation microplanning in Northern Nigeria. BMJ Global Health 2019; 4:e001606.

Artigo apresentado em 28/01/2021

Aprovado em 12/02/2021

Versão final apresentada em 14/02/2021

Editores-chefes: Romeu Gomes, Antônio Augusto Moura da Silva 